\title{
Erratum: Erosive Esophagitis after Bariatric Surgery: Banded Vertical Gastrectomy versus Banded Roux-en-Y Gastric Bypass
}

Gustavo Peixoto Soares Miguel • João Luiz Moreira Coutinho Azevedo •

Paulo Henrique Oliveira de Souza • João de Siqueira Neto • Felipe Mustafa •

Évelyn Saiter Zambrana • Perseu Seixas de Carvalho

Published online: 12 January 2011

(C) Springer Science+Business Media, LLC 2011

Erratum to: Obes Surg

DOI 10.1007/s11695-010-0321-x

The original publication should not have included Figure 1the image and reference thereto should be disregarded.

The online version of the original article can be found at http://dx.doi. org/10.1007/s11695-010-0321-x.

G. P. S. Miguel $(\bowtie) \cdot$ P. H. O. de Souza $\cdot$ J. de Siqueira Neto •

F. Mustafa - É. S. Zambrana • P. S. de Carvalho

Federal University of Espírito Santo-UFES,

Vitória, ES, Brazil

e-mail: gsoaresp@terra.com.br

J. Luiz Moreira Coutinho Azevedo

Federal University of São Paulo-Unifesp,

São Paulo, SP, Brazil 